\title{
Relapsing IgG4-related orbital inflammatory pseudotumor after achieving success with rituximab
}

\begin{abstract}
IgG4-related disease (IgG4-RD) is a disease of fibroinflammatory with infiltration of IgG4-positive plasma cells. The lesions show focal or diffuse nodular formations in one or multiple organs; due to the infiltration of lymphocytes and plasma cells with fibrosis. The IgG4-DR is recognized as an important cause of idiopathic inflammatory disease that must be differentiated with other pathologies. The measurement of normal lgG4 levels is not enough to exclude IgG4-RD. The treatment covers the symptomatic aspect and use of systemic corticosteroids (CS), and immunomodulatory and biological therapy. The relapsing is might be misdiagnosis. We propose to consider the imaging follow-up for the therapeutic management
\end{abstract}

Keywords: pseudotumor • rituximab • exophthalmos

\section{Introduction}

IgG4-related disease (IgG4-RD) is an emerging disease of fibroinflammatory nature, infiltration of IgG4-positive plasma cells [1]. The multiorgan involvement has a histopathological connection as well as the immunohistochemical staining pattern [2]. The lesions show focal or diffuse nodular formations in one or multiple organs; due to the infiltration of lymphocytes and plasma cells with fibrosis [3] in a pattern called "storiform" fibrosis, a tendency to affect veins (obliterative phlebitis); and mild to moderate tissue eosinophilia [4]. Currently, IgG4-DR is recognized as an important cause of idiopathic inflammatory disease that must be differentiated with other pathologies that include Hodgkin lymphoma and non-Hodgkin's lymphoma, granulomatosis with polyangiitis (Wegener's), Graves' orbitopathy, and other conditions [5], their incidence was estimated at 4.7\%$6.3 \%$ of all orbital diseases [6]. The proposed nomenclature for 2012 for this disorder is IgG4related ophthalmic disease because the disease can extend beyond the orbit comprising extrinsic muscles and trigeminal nerve among others; when the soft tissue orbital compromises it is termed IgG4-related orbital inflammation (or IgG4-related orbital inflammatory pseudotumor) [7]. The bilateral location of the pseudotumor in adult patients would suggest the presence of generalized disease such as vasculitis or generalized lymphoid hyperplasia. On the other hand, the measurement of normal IgG4 levels is not enough to exclude IgG4-RD nor should its diagnosis be considered if it is elevated [7]. In relation to treatment, there are approaches that cover the symptomatic aspect, use of systemic corticosteroids (CS), use of immunomodulatory and biological therapy [8] systemic or local.

\section{Case presentation}

A 20-year-old man presented with progressive exophthalmos of the right eye (RY) of one year of evolution characterized by ocular pain, diminution of visual acuity, ipsilateral temporal headache, malaise and respiratory distress due to progressive obstruction of the left nostril. $\mathrm{He}$ went to a first medical center where a tumor biopsy was performed on the left nasal mucosa whose microscopic report included stroma with edema and moderate inflammatory infiltrate characterized by the presence of lymphocytes, plasma cells and few eosinophils; without granulomas description or vasculitis characteristics. Immunohistochemistry wasn't performed. The right eyeball tumor biopsy showed irregular dense connective tissue fibrosis and lymphocytic inflammatory infiltrate with formation of lymphoid nodules. The initial diagnosis was allergic polyposis and chronic inflammatory follicular "process" associated with fibrosis. The initial management that I was receiving was based on prednisone $1 \mathrm{mg}$ / $\mathrm{Kg}$ weight for 5 months and brimonidine improvement and appearance of adverse effects by CS came to our medical center. The clinical examination showed diminished visual acuity, IOP $15 \mathrm{mmHg}$ (both eyes), exophthalmos and limitation of right eye abduction. Laboratory tests showed normal blood count, kidney and liver function; C-reactive protein was elevated 12 $\mathrm{mg} / \mathrm{L}(<8.2 \mathrm{mg} / \mathrm{dL})$, as was sedimentation rate 14 $\mathrm{mm} / \mathrm{h}$. TSH, thyroxine, anti-TPO, rheumatoid the mucous glands proliferated without atypia, $0.2 \%$ for 2 months. Due to the poor clinical
Oscar Vega-Hinojosa*1, Berioska Manzaneda $\mathbf{M}^{2}$, Lisette M. Bedoya $M^{3}$ \& Martin Sangüeza $A^{4}$

'Research Unit Reumacenter Clinic, Juliaca, Peru

${ }^{2}$ Unit of Ophthalmology Hospital-III, Essalud Juliaca, Universidad Católica de Santa María, Arequipa, Peru

${ }^{3}$ Department of Radiology Hospital-III, Essalud Juliaca, Peru

${ }^{4}$ Pathology and Molecular Biology Office, La Paz, Bolivia

*Author for correspondence: vegalines@hotmail.com 
factor, anti-CCP, FANA screen, perinuclear and cytoplasmatic ANCA, bacilloscopy for $\mathrm{BK}$, profile ENA were negative. Total IgG was normal $1250 \mathrm{mg} / \mathrm{dL}$ (normal 650-1600) and IgG4 $150 \mathrm{mg} / \mathrm{dL}(<135)$. MRI was consistent with inflammation lesion periorbital. Based on Comprehensive diagnostic criteria for IgG4-related disease, 2011 and 2012 [9-11], IgG4-RD definite was diagnosed. Therefore, induction treatment with methylprednisolone followed with rituximab $1 \mathrm{~g}$ and repeated in 15 days. After one month, the patient reported remission of symptoms, normalization of IOP and decrease in total IgG levels $1040 \mathrm{mg} / \mathrm{dL}$ and IgG4 $70 \mathrm{mg} / \mathrm{dL}$; however due to persistent proptosis it was decided to add azathioprine (AZA) $2 \mathrm{mg} / \mathrm{Kg}$ weight. During the followup period, bad adherence was reported to the AZA indications until it was abandoned. After two and a half years the patient reported pain to eye movements, headache and respiratory distress, IOP examination of $14 \mathrm{~mm} \mathrm{Hg}$ in both eyes, visual acuity preserved, moderated limitation in abduction and exophthalmometry of $24 \mathrm{~mm}$ in the right eye and $18 \mathrm{~mm}$ in the left eye, an irregular mass of rapid growth under the right upper eyelid and new masses reported in MRI; Total IgG levels were $1689 \mathrm{mg} / \mathrm{dL}$ and IgG4 $70 \mathrm{mg} / \mathrm{dL}$, with the previous therapy being reinitiated. After 20 days of restarting the treatment, eye movements were normal; exophthalmometry of 22 and $18 \mathrm{~mm}$ in right and left eye respectively. IOP of $13 \mathrm{mmHg}$ in both eyes, tumor mass that compromises the persistent lacrimal gland below the right upper eyelid (Figures 1-3).

\section{Discussion}

The percentage of recurrence reaches $10 \%$ in autoimmune pancreatitis [12]; however, the percentage and time range of recurrence of symptoms and the presence of a "new" pseudotumor is scarcely reported and in those mentioned recurrence occurs at two years [13]; On the other hand, serum IgG4 levels would not necessarily be elevated in relapses [14]. The levels of IgG4 had a total IgG4 / IgG ratio of $12 \%$, which decreased to $6.73 \%$ at one month of treatment and during the relapse reached $4.7 \%$. It has been recognized that there is no synchronism between the appearances of systemic or localized lesions; but if there would be at the time of development of eye injuries; one report has indicated that elevated levels of IgG4 (> $900 \mathrm{mg} / \mathrm{dL})$ would be associated with concurrent orbital and extraorbital lesions [15]. Corticosteroids provoke a dramatic response at 48 hours after systemic administration especially
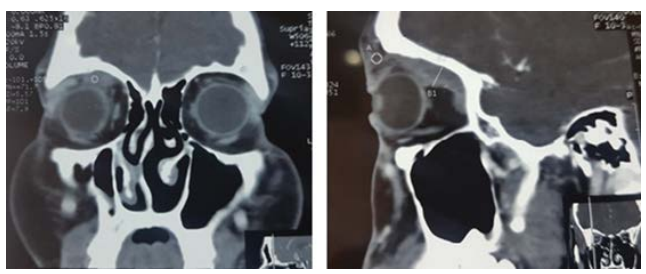

Figure 1. Initial computed tomography (2016) Coronal and sagital view showing proliferation in superior and lateral rectus muscles, and lacrimal gland of the right orbit.
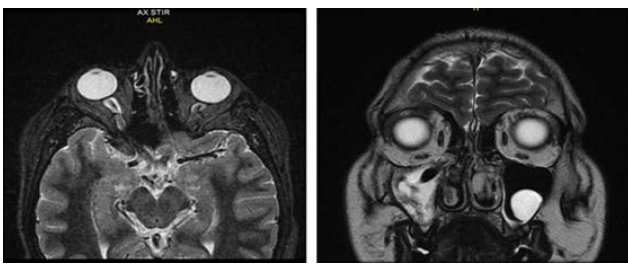

Figure 2. MRI control; Coronal view showing thickening the retroconal fat and optic nerve and mild proptosis; Axial view also shows sinusitis.

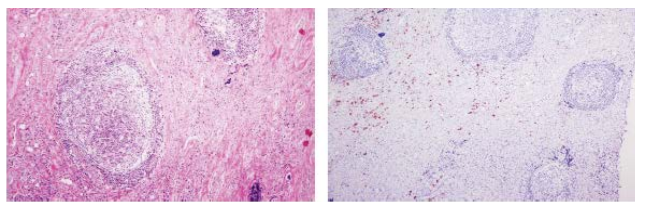

Figure 3. (A) Follicular chronic dacryoadenitis; Lobules of lacrimal glands atrophied by chronic mononuclear inflammatory infiltrate, presence of lymphoid follicles; HE; increase 100X; (B) Immunostaining for IgG4 shows IgG4+ plasma cells.

in cases of idiopathic orbital myositis in cases of non-remission-myositis IOI shows a low positive predictive value and on the other hand a response should not be confused with a rebound after tapering the CS [16].

Corticosteroids are first-line drugs that can be replaced with AZA, mycophenolate mofetil, and methotrexate; those who were not evaluated in clinical trials, are used frequently as CS sparing agents or remission-maintenance drugs after CSinduced remissions. Rituximab is indicated for patients with recurrent or refractory disease, levels of IgG4 decline rapidly while levels of other IgG subclasses remain stable $[17,18]$. Unlike IgG4related Sclerosing Cholangitis in which elevated alkaline phosphatase levels are a predictor of relapse after RTX induction therapy in the IgG4$\mathrm{RD}$, predictive marker isn't known [19,20]; on the other hand the elevation of pretreatment levels of IgG4, immunoglobulin E (IgE) and blood eosinophil concentrations independently predicted future relapses [20]. The objective of the treatment of IgG4-RD is to alleviate symptoms and prevent disease-related complications and irreversible fibrosis [21]. Some studies have suggested its controversial association [22,23] 
with the presence of association of IgG4-RD with increased risk of malignancy, particularly at one year of diagnosis. The modulation of $\mathrm{B}$ cells and plasma cells offers good therapeutic effects. Currently, a chemotherapeutic agent, like bortezomib, is also in use [24]; its administration concomitant with cyclophosphamide produced the success of efficient depletion of long-lived plasma cells [25].

\section{Conclusion}

The IgG4-RD orbital inflammatory pseudotumor apparently has low prevalence; however, monitoring of serum IgG4 levels doesn't necessarily indicate the state of the disease, so follow-up by imaging should be considered in order to suspend treatment.

Conflict of interest

None

Funding

None

Acknowledgement

Mr. Brian A. Loza-Calderon by translate.

\section{References}

1. Kamisawa T, Funata N, Hayashi Y et al. Close relationship between autoimmune pancreatitis and multifocal fibrosclerosis. Gut. 52(5), 683-687 (2003).

2. Cheuk W, Chan JK: IgG4-related sclerosing disease: a critical appraisal of an evolving clinicopathologic entity. Adv. Anat. Pathol. 17(5), 303-332 (2010).

3. Uehara T, Hamano H, Kawa $S$ et al. Chronic gastritis in the setting of autoimmune pancreatitis. Am. J. Surg. Pathol. 34(9), 1241-1249 (2010).

4. Deshpande V, Zen Y, Chan JK et al. Consensus statement on the pathology of IgG4-related disease. Mod. Pathol. 25(9), 1181-1192 (2012).

5. Sato Y, Ohshima K, Ichimura K et al. Ocular adnexal IgG4-related disease has uniform clinicopathology. Pathol. Int. 58(8), 465-470 (2008).

6. Plaza JA, Garrity JA, Dogan A et al. Orbital inflammation with IgG4-positive plasma cells: manifestation of IgG4 systemic disease. Arch. Ophthalmol. 129(4), 421-428 (2011).

7. Stone JH, Khosroshahi A, Deshpande $\mathrm{V}$ et al. Recommendations for the nomenclature of IgG4-related disease and its individual organ system manifestations. Arthritis. Rheum. 64(10), 3061-3067 (2012).

8. Savino G, Battendieri R, Siniscalco A et al. Intraorbital injection of Rituximab in idiopathic orbital inflammatory syndrome: case reports. Rheumatol. Int. 35(1), 183-188 (2015).

9. Goto H, Takahira M, Azumi A. Japanese Study Group for Ig GROD: Diagnostic criteria for IgG4-related ophthalmic disease. Jpn. J. Ophthalmol. 59(1), 1-7 (2015).
10. Umehara H, Okazaki K, Masaki Y et al. Comprehensive diagnostic criteria for IgG4-related disease (IgG4-RD), 2011. Mod. Rheumatol. 22(1), 21-30 (2012).

11. Umehara H, Okazaki K, Nakamura T et al. Current approach to the diagnosis of IgG4-related disease Combination of comprehensive diagnostic and organspecific criteria. Mod. Rheumatol. 27(3), 381-391 (2017).

12. Kamisawa T, Shimosegawa T, Okazaki K et al. Standard steroid treatment for autoimmune pancreatitis. Gut. 58(11), 1504-1507 (2009).

13. Bergmann KR, Schloff S, Schiff J. Contralateral recurrence of idiopathic orbital inflammatory syndrome in a pediatric patient. Minn. Med. 97(10), 46-47 (2014).

14. Ghazale A, Chari ST, Zhang L et al. Immunoglobulin G4-associated cholangitis: clinical profile and response to therapy. Gastroenterology. 134(3), 706-715 (2008).

15. Kubota T, Katayama M, Moritani $S$ et al. Serologic factors in early relapse of IgG4-related orbital inflammation after steroid treatment. Am. J. Ophthalmol. 155(2), 373-379 e371 (2013).

16. Mombaerts I, Bilyk JR, Rose GE et al. Expert Panel of the Orbital S: Consensus on Diagnostic Criteria of Idiopathic Orbital Inflammation Using a Modified Delphi Approach. JAMA. Ophthalmol. 135(7), 769-776 (2017).

17. Khosroshahi A, Carruthers MN, Deshpande V et al. Rituximab for the treatment of IgG4-related disease: lessons from 10 consecutive patients. Medicine. (Baltimore) 91(1), 57-66 (2012).

18. Khosroshahi A, Bloch DB, Deshpande V et al. Rituximab therapy leads to rapid decline of serum IgG4 levels and prompt clinical improvement in IgG4-related systemic disease. Arthritis. Rheum. 62(6), 1755-1762 (2010).

19. Majumder S, Mohapatra S, Lennon RJ et al. Rituximab maintenance therapy reduces rate of relapse of pancreaticobiliary immunoglobulin G4-related disease. Clin. Gastroenterol. Hepatol. 16(12), 1947-1953 (2018).

20. Wallace ZS, Mattoo H, Mahajan VS et al. Predictors of disease relapse in IgG4-related disease following rituximab. Rheumatology (Oxford). 55(6), 1000-1008 (2016).

21. Khosroshahi A, Wallace ZS, Crowe JL et al. International consensus guidance statement on the management and treatment of IgG4-related disease. Arthritis. Rheumatol. 67(7), 1688-1699 (2015).

22. Huggett MT, Culver EL, Kumar $M$ et al. Type 1 autoimmune pancreatitis and IgG4-related sclerosing cholangitis is associated with extrapancreatic organ failure, malignancy, and mortality in a prospective UK cohort. Am. J. Gastroenterol. 109(10), 1675-1683 (2014).

23. Shiokawa M, Kodama Y, Yoshimura K et al. Risk of cancer in patients with autoimmune pancreatitis. Am. J. Gastroenterol. 108(4), 610-617 (2013).

24. Oles K, Szczepanski W, Skladzien J et al. IgG4-related inflammatory orbital pseudotumors - a retrospective case series. Folia. Neuropathol. 53(2), 111-120 (2015).

25. Ri M. Mechanism of action and determinants of sensitivity to the proteasome inhibitor bortezomib in multiple myeloma therapy. Rinsho. Ketsueki. 57(5), 537-545 (2016). 